\title{
Long-Term Potentiation Induced by $\theta$ Frequency Stimulation Is Regulated by a Protein Phosphatase-1-Operated Gate
}

\author{
George P. Brown, ${ }^{1}$ Robert D. Blitzer, ${ }^{2,3}$ John H. Connor, ${ }^{4}$ Tony Wong, ${ }^{2}$ Shirish Shenolikar, ${ }^{4}$ Ravi lyengar, ${ }^{1}$ and \\ Emmanuel M. Landau ${ }^{1-3}$
}

Departments of ${ }^{1}$ Pharmacology and 2 Psychiatry, Mount Sinai School of Medicine, New York, New York 10029, ${ }^{3}$ Psychiatry

Service, Bronx Veterans Administration Medical Center, Bronx, New York 10468, and ${ }^{4}$ Department of Pharmacology and

Cancer Biology, Duke University Medical Center, Durham, North Carolina 27712

Long-term potentiation (LTP) can be induced in the Schaffer collateral $\rightarrow$ CA1 synapse of hippocampus by stimulation in the $\theta$ frequency range $(5-12 \mathrm{~Hz})$, an effect that depends on activation of the CAMP pathway. We investigated the mechanisms of the cAMP contribution to this form of LTP in the rat hippocampal slice preparation. $\theta$ pulse stimulation (TPS; 150 stimuli at $10 \mathrm{~Hz}$ ) by itself did not induce LTP, but the addition of either the $\beta$-adrenergic agonist isoproterenol or the cAMP analog 8-bromoCAMP (8-Br-cAMP) enabled TPS-induced LTP. The isoproterenol effect was blocked by postsynaptic inhibition of CAMPdependent protein kinase. Several lines of evidence indicated that CAMP enabled LTP by blocking postsynaptic protein phosphatase-1 (PP1). Activators of the cAMP pathway reduced PP1 activity in the CA1 region and increased the active form of inhibitor-1, an endogenous inhibitor of PP1. Postsynaptic injection of activated inhibitor-1 mimicked the LTP-enabling effect of
cAMP pathway stimulation. TPS evoked complex spiking when isoproterenol was present. However, complex spiking was not sufficient to enable TPS-induced LTP, which additionally required the inhibition of postsynaptic PP1. PP1 inhibition seems to promote the activation of $\mathrm{Ca}^{2+} /$ calmodulin-dependent protein kinase (CaMKII), because (1) a CaMKII inhibitor blocked the induction of LTP by TPS paired with either isoproterenol or activated inhibitor-1 and (2) CaMKII in area CA1 was activated by the combination of TPS and 8-Br-cAMP but not by either stimulus alone. These results indicate that the cAMP pathway enables TPS-induced LTP by inhibiting PP1, thereby enhancing $\mathrm{Ca}^{2+}$ independent CaMKII activity.

Key words: LTP induction; cAMP-dependent protein kinase; inhibitor-1; protein phosphatase-1; $\mathrm{Ca}^{2+} /$ calmodulin-dependent protein kinase; gating; complex spikes
Many synapses in the CNS can be strengthened or weakened by repetitive stimulation. This phenomenon has been intensively studied in the rodent hippocampus, particularly at the synapse between the pyramidal cells of areas CA 3 and CA1 (Bliss and Collingridge, 1993; Malenka and Nicoll, 1999), and many of these studies have investigated how particular patterns of stimulation give rise to different forms of synaptic plasticity. Such factors as the frequency, intensity, and duration of the stimulation determine whether the synapse is strengthened or weakened, as well as the persistence of the synaptic change. Remarkably, even when two different patterns induce seemingly similar synaptic changes, they may do so via different molecular mechanisms. Efforts to understand the role of the pattern of stimulation in synaptic plasticity may prove particularly valuable, because pronounced spontaneous frequency variations characterize hippocampal neuronal activity in behaving animals (Fenton and Muller, 1998). Studies in hippocampal slices have established that the pattern of stimulation determines the mechanism of synaptic change, so it is reasonable to speculate that the natural patterns of the hippocampus in vivo may give rise to multiple forms of synaptic plasticity.

An important principle of pattern-dependent plasticity is the relationship between stimulus frequency and the direction of synaptic change (Dudek and Bear, 1992; Katsuki et al., 1997). In slices of rat hippocampus, low-frequency stimulation (1-3 Hz; LFS) produces a long-term depression of synaptic transmission (LTD).

Received June 26, 2000; revised Aug. 10, 2000; accepted Aug. 14, 2000.

This research was supported by National Institutes of Health Grants NS33646 to E.M.L., GM5408 to R.I., and DK5204 to S.S. and a Veterans Administration Merit Award to E.M.L. G.P.B. is a recipient of individual National Research Service Award GM 18887. We thank H. Schulman and A. P. Braun for samples of autocamtide-3 and control peptide.

Correspondence should be addressed to Dr. Robert D. Blitzer, Box 1215, Department of Pharmacology, Mount Sinai School of Medicine, One Gustave L. Levy Place, New York, NY 10029. E-mail: rb2@doc.mssm.edu.

Copyright (C) 2000 Society for Neuroscience $0270-6474 / 00 / 207880-08 \$ 15.00 / 0$
Conversely, long-term potentiation (LTP) is induced when stimulation is delivered at higher frequencies $(30-100 \mathrm{~Hz}$; HFS). The crossover or neutral frequency, at which repetitive stimulation does not modify synaptic strength, is $\sim 10 \mathrm{~Hz}$ at this synapse. However, the relationship between the stimulation frequency and the direction of the change in synaptic strength is sensitive to certain pharmacological manipulations, including the inhibition of protein kinases or phosphatases and the activation of $\beta$-adrenergic receptors (Coussens and Teyler, 1996; Thomas et al., 1996; Katsuki et al., 1997). In both mouse and rat hippocampal slices, LTP can be induced by stimulation at the neutral frequency in the presence of a $\beta$-adrenoceptor agonist, an effect that is mediated by the cAMPsignaling pathway (Thomas et al., 1996).

This pharmacological modification of the relationship between pattern and LTP touches on a relatively unexplored issue in LTP research. The intact hippocampus is richly innervated by other nuclei, such as the noradrenergic locus ceruleus. These extrinsic inputs may provide another level of control over hippocampal synaptic plasticity, in addition to any intrinsic variations in the frequency of hippocampal activity. Under certain conditions extrinsic input could be a major determinant of synaptic change, such as when a synapse is being activated at a rate near the neutral frequency. The slice data suggest that an increase in noradrenergic input could induce LTP at such synapses. In the behaving rat, a predominant component of hippocampal activity occurs near the neutral frequency, in the range of $5-12 \mathrm{~Hz}$ ( $\theta$ frequency). Therefore, variations in noradrenergic tone may exert an important influence on synaptic plasticity in vivo.

Most investigations of LTP in hippocampal slices have used multiple trains of HFS for induction, usually delivered at $100 \mathrm{~Hz}$. We and others have shown a requirement for the cAMP pathway in HFS-induced LTP and have demonstrated the existence of a postsynaptic gating mechanism that regulates the induction of LTP (Frey et al., 1993; Blitzer et al., 1995, 1998; Otmakhova et al., 2000). 
The gating mechanism comprises two antagonistic pathways. The cAMP arm favors LTP induction and includes adenylyl cyclase, cAMP, and cAMP-dependent kinase (PKA). Opposing the cAMP pathway is calcineurin, a $\mathrm{Ca}^{2+} /$ calmodulin-activated protein phosphatase. These pathways converge on the regulatory protein inhibitor-1, which in its phosphorylated form inhibits protein phosphatase- 1 (PP1). The postsynaptic $\mathrm{Ca}^{2+}$ entry that accompanies HFS is thought to activate both arms of the gating mechanism, as well as the LTP-signaling pathway that begins with $\mathrm{Ca}^{2+}$ calmodulin-dependent protein kinase (CaMKII) (Lisman, 1994). The same gating mechanism might explain how $\beta$-adrenergic stimulation enables neutral frequency stimulation to induce LTP. The source of postsynaptic cAMP in this case would be $\beta$-receptoractivated adenylyl cyclases, whereas the $\mathrm{Ca}^{2+}$ influx associated with $\theta$ frequency stimulation would activate both calcineurin and CaMKII.

The cAMP pathway influences the behavior of many membrane channels and signaling systems, so it is likely to contribute to synaptic plasticity in ways that are independent of the PP1 gate. Recent work in mouse hippocampus has shown a cAMP-mediated increase in postsynaptic excitability that is important in $\theta$ pulse stimulation (TPS)-induced LTP (Thomas et al., 1998; Winder et al., 1999). These studies establish that TPS must evoke postsynaptic burst spiking for LTP to occur and that $\beta$-adrenergic stimulation enhances this activity. The mechanism underlying this effect of the cAMP pathway is not well understood, but it may be mediated by an increase in the activity of MAP kinase (ERK). The present study was conducted to investigate the mechanisms underlying the contribution of the cAMP pathway to TPS-induced LTP.

\section{MATERIALS AND METHODS}

Electrophysiology. Dissection and recording methods were similar to those described previously (Blitzer et al., 1995). Intracellular recordings of area CA1 neurons from male Sprague Dawley rats (125-200 gm) were obtained in a submersion chamber at $31^{\circ} \mathrm{C}$. Slices were superfused with a solution containing (in mM) $\mathrm{NaCl}$ (118), $\mathrm{KCl}$ (3.5), $\mathrm{MgSO}_{4}$ (1.3), $\mathrm{CaCl}_{2}$ (3.5), $\mathrm{NaH}_{2} \mathrm{PO}_{4}(1.25), \mathrm{NaHCO}_{3}(24)$, and glucose (15) and bubbled with $95 \%$ $\mathrm{O}_{2} / 5 \% \quad \mathrm{CO}_{2}$. Cells in the stratum pyramidale were impaled with sharp electrodes containing $3 \mathrm{M} \mathrm{KCl}\left(R_{e}=60-90 \mathrm{M} \Omega\right)$, and field recordings were made with electrodes $\left(2 \mathrm{M} \mathrm{NaCl} ; R_{e}=2-5 \mathrm{M} \Omega\right)$ placed in the stratum radiatum. Isoproterenol, 2-amino-5-phosphonovaleric acid (APV), and 8-bromo-cAMP (8-Br-cAMP) were applied in the superfusate. Thiophosphorylated inhibitor-1 (I-1), recombinant T35A nonphosphorylatable I-1, autocamtide-3, its control peptide (gifts of A. P. Braun and H. Schulman), and the $\mathrm{Rp}$ isomer of cyclic adenosine-3',5'-monophosphothioate (RpcAMPS) were included in the intracellular electrode and allowed to diff use into the cell; in these experiments, TPS was delivered 40 min after impalement. For synaptic stimulation, monophasic, constant-current pulses (100 $\mu \mathrm{sec}$ in duration) were delivered to the Schaffer collaterals (stratum radiatum in area CA3). Test pulses were usually delivered in series of four pulses ( $5 \mathrm{sec}$ apart), with the series separated by $5 \mathrm{~min}$, and the EPSPs within each series were averaged. For some experiments (see Fig. 5), test stimuli were given every $30 \mathrm{sec}$, and the EPSPs were not averaged. EPSP amplitude and maximum initial slope (defined as the greatest slope within any $1 \mathrm{msec}$ interval between the stimulus artifact and the EPSP peak) were measured either off-line for the averaged waveforms or on-line for the individual EPSPs in some experiments (see Fig. 5). TPS consisted of a single train of 150 stimuli delivered at $10 \mathrm{~Hz}$. For most experiments that included intracellular recording, the stimulus intensity was adjusted to produce a $10-15 \mathrm{mV}$ intracellular EPSP. However, in experiments designed to induce repetitive postsynaptic spiking (see Fig. 5), a stronger stimulus was used during TPS, sufficient to evoke single spikes reliably during pre-TPS test pulses. This stimulus was approximately twice as strong as that used during TPS in the other experiments. For those experiments using only field recording, the stimulus during TPS was adjusted to evoke a $1 \mathrm{mV}$ field potential during test pulses. Data were analyzed by Student's $t$ test or by ANOVAs followed by Newman-Keuls post hoc comparisons. Summary data are presented as group means with SE bars.

CaMKII activity. Hippocampal slices were incubated with 8-Br-cAMP for $2-5 \mathrm{hr}$ before recording. Control slices were stimulated with TPS alone, 8-Br-cAMP alone, or neither. The slices were removed from the recording chamber and placed on a cold plate within $2-3$ min after TPS, and the CA1 region was dissected out and frozen at $-70^{\circ} \mathrm{C}$. Extracts from individual CA1 regions were assayed for CaMKII activity essentially as described (Mayford et al., 1995). Tissue was homogenized in a buffer containing 50 mM HEPES, pH 7.5, 0.5 mM EGTA, $0.5 \mathrm{~mm}$ EDTA, $15 \mathrm{~mm}$ sodium pyrophosphate, $25 \mathrm{~mm} \mathrm{NaF}, 75 \mu \mathrm{g} / \mathrm{ml}$ leupeptin, $0.1 \mathrm{mg} / \mathrm{ml}$ aprotinin, 0.4 mM DTT, and 0.1 mM PMSF. Protein levels were assayed by Lowry assay using BSA as the standard. The enzyme reaction mix consisted of $50 \mathrm{~mm}$
HEPES, pH 7.5, 10 mm $\mathrm{MgCl}_{2}, 100 \mu \mathrm{g} / \mathrm{ml}$ BSA, $200 \mu \mathrm{g} / \mathrm{ml}$ leupeptin, 0.4 mM DTT, 0.6 mM EGTA, 0.2 mM EGTA, $2 \mu \mathrm{M}$ Wiptide (PKA inhibitor; American Peptide Co.), $2 \mu \mathrm{M}$ PKC (19-36) peptide (PKC inhibitor; American Peptide Co.), $200 \mu \mathrm{M}$ ATP, $100 \mu \mathrm{Ci} / \mathrm{ml}\left[\gamma^{-32} \mathrm{P}\right] \mathrm{ATP}$, and $20 \mu \mathrm{M}$ autocamtide II (Calbiochem, La Jolla, CA), with either $1 \mathrm{~mm} \mathrm{CaCl}$ and $5 \mu \mathrm{g} / \mathrm{ml}$ calmodulin (total activity) or $2 \mathrm{mM}$ EGTA $\left(\mathrm{Ca}^{2+}\right.$-independent activity). Enzyme reactions were performed in quadruplicate at $30^{\circ} \mathrm{C}$ for 1 min in a final volume of $50 \mu \mathrm{l}$. The reaction was initiated by the addition of $2 \mu \mathrm{g}$ of CA1 extract and terminated by the addition of an equal volume of $10 \%$ ice-cold TCA. Protein was pelleted, and the supernatant was spotted onto Whatman P81 filter paper and washed three times for $5 \mathrm{~min}$ with water. The amount of ${ }^{32} \mathrm{P}$ incorporated into substrate peptide was determined by liquid scintillation counting.

Inhibitor-1 immunoblotting. Hippocampal slices were placed in a submersion chamber bubbled with $95 \% \mathrm{O}_{2} / 5 \% \mathrm{CO}_{2}$, at room temperature, and were exposed to either 8-Br-cAMP (1 mM for $>2 \mathrm{hr}$ ) or isoproterenol (1 $\mu \mathrm{M}$ for $10 \mathrm{~min})$. Immediately after treatment, the slices were frozen, and the CA1 region was dissected out and stored at $-70^{\circ} \mathrm{C}$. The tissue samples were lysed in $100 \mu$ l of lysis buffer $(50 \mathrm{~mm}$ Tris, $4 \mathrm{~mm}$ EGTA, $10 \mathrm{~mm}$ EDTA, $15 \mathrm{~mm}$ Na phosphate, $100 \mathrm{~mm} \beta$-glycerol phosphate, $10 \mathrm{~mm} \mathrm{NaF}$, $0.1 \mathrm{~mm}$ pepstatin, $1 \mathrm{~mm}$ PMSF, and $2 \mathrm{~mm}$ benzamidine, $\mathrm{pH} 7.5$ ) and ground three times for $30 \mathrm{sec}$ each with a pellet pestle (Kontes glassware). The resulting lysate was spun down at $15,000 \times g$ at $4^{\circ} \mathrm{C}$ for $10 \mathrm{~min}$, and the supernatant was then assayed for total protein concentration. Twenty micrograms of protein from each sample were run on a $15 \%$ SDS-PAGE gel. The samples were transferred onto a polyvinylidene difluoride membrane overnight (25 A constant current) and then blotted with an antibody to either phosphorylated DARPP-32/I-1 or recombinant human I-1. The monoclonal antibody to phosphorylated DARPP-32/I-1 was provided by G. L. Snyder and P. Greengard (Snyder et al., 1992). Both blots were developed using ECL and then digitized with a densitometer and analyzed using ImageQuant.

Protein phosphatase-1 activity. Hippocampal slices were treated and dissected as described for the I-1 immunoblot (above). Individual CA1 regions were homogenized in $50 \mathrm{~mm}$ Tris, pH 7.5, $0.2 \mathrm{~mm}$ EDTA, $2 \mu \mathrm{g} / \mathrm{ml}$ leupeptin, $2 \mu \mathrm{g} / \mathrm{ml}$ aprotinin, and $10 \mathrm{~nm}$ okadaic acid. Phosphatase activity of $20 \mathrm{ng}$ of CA1 extract was measured with the Protein Phosphatase Assay System (Life Technologies, Gaithersburg, MD) in a final concentration of $3.3 \mathrm{~nm}$ okadaic acid. Each group was run in quadruplicate. By the use of this method, inclusion of $100 \mathrm{nM}$ thiophosphorylated I-1 inhibited $>85 \%$ of phosphatase activity, confirming the selectivity of the system for PP1.

\section{RESULTS}

\section{TPS-induced LTP requires the postsynaptic cAMP pathway}

The initial experiments were designed to establish the conditions for inducing LTP in adult rat hippocampal slices using TPS and $\beta$-adrenergic stimulation (TPS-LTP). The combination of TPS (150 stimuli delivered to the Schaffer collaterals at $10 \mathrm{~Hz}$ ) and isoproterenol $(1 \mu \mathrm{M})$ reliably produced LTP (Fig. $1 A, B$; intracellular EPSP slope $=173 \pm 8 \%$ of baseline at $30 \mathrm{~min}$ ). During isoproterenol application, the EPSP was depressed in some slices; however, no persistent effect of isoproterenol alone was observed after $30 \mathrm{~min}$ of washout (Fig. $1 \mathrm{~B}$ ). When isoproterenol was omitted, there was a nonsignificant tendency for TPS alone to depress synaptic transmission (intracellular slope $=85 \pm 11 \%$ of baseline at $30 \mathrm{~min} ; n=6$ ).

$\beta$-Adrenergic stimulation increases neuronal cAMP production in the CA1 region, and postsynaptic effects of $\beta$-adrenergic agonists have been attributed to increased cAMP in CA1 pyramidal cells (Segal et al., 1981; Madison and Nicoll, 1986; Pedarzani and Storm, 1993). If the $\beta$-adrenergic contribution to TPS-induced LTP (TPSLTP) is also mediated by the cAMP-dependent inhibition of PP1, then direct activation of the cAMP pathway should also enable TPS-LTP. This prediction was tested by delivering TPS in the presence of the membrane-permeable analog 8-Br-cAMP. By itself, 8-Br-cAMP had no effect on synaptic efficiency $(97 \pm 6 \%$ at 30 $\min ; n=3)$. However, 8 -Br-cAMP enabled TPS to induce LTP (Fig. $1 C ; 156 \pm 12 \%$ at $30 \mathrm{~min} ; n=7$ ).

The cAMP pathway exerts both presynaptic and postsynaptic effects that are likely to influence synaptic plasticity at the CA3 $\rightarrow$ CA1 synapse (Madison and Nicoll, 1986; Ma et al., 1999). To determine whether the LTP-promoting effect of cAMP pathway activation was mediated by the postsynaptic cAMP pathway, we included the inhibitory cAMP analog Rp-cAMPS in the intracellular electrode and stimulated with TPS and isoproterenol. In these cells, the synaptic response was potentiated only transiently and returned to near-baseline levels within $30 \mathrm{~min}$. Thus, activation of 
$A_{1}$

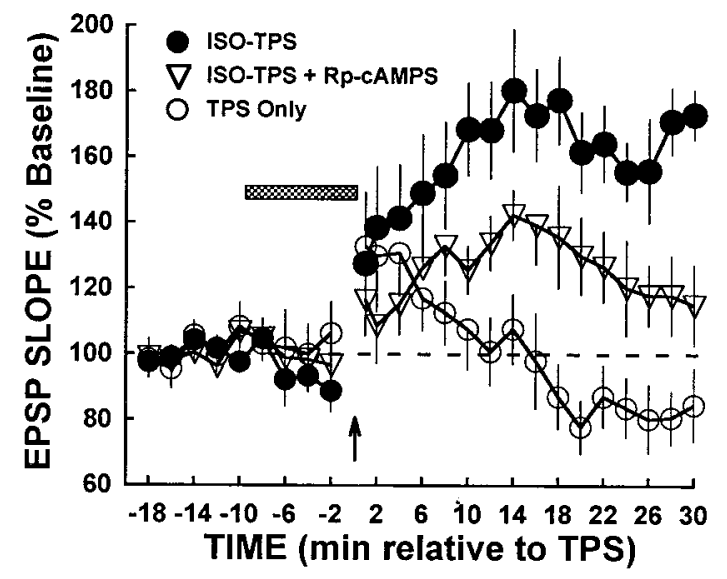

C

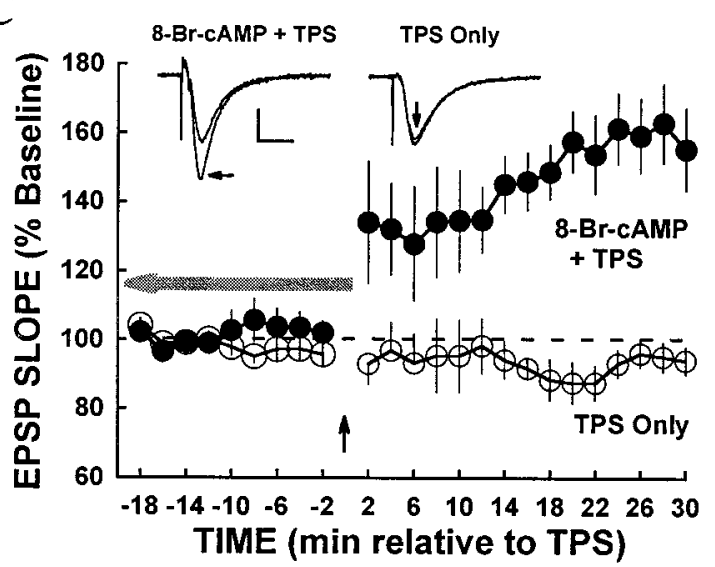

$A_{2}$

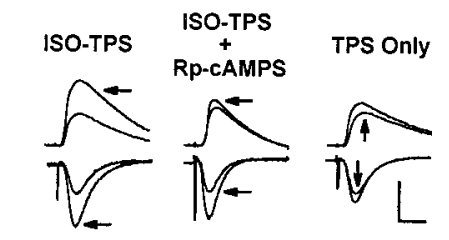

B

D

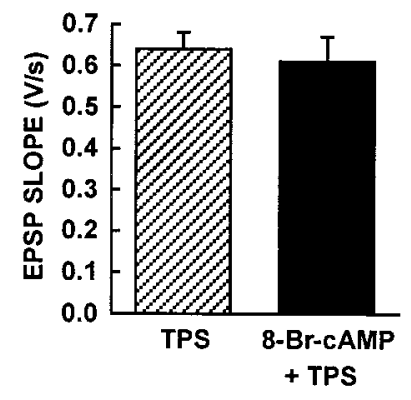

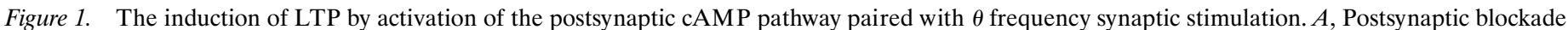

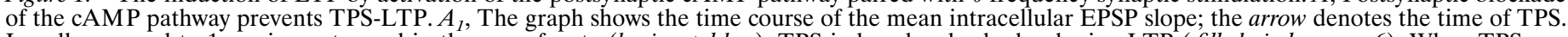

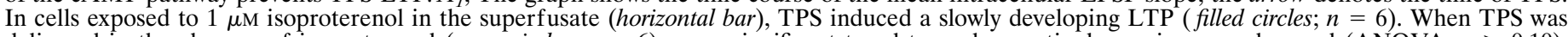

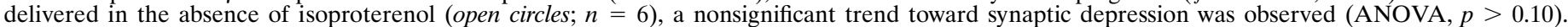

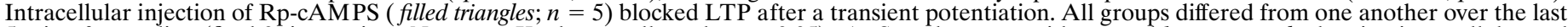

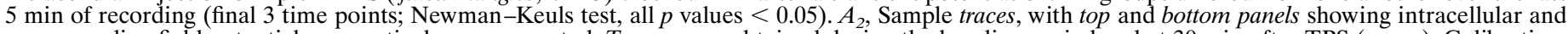

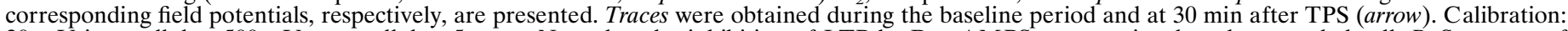

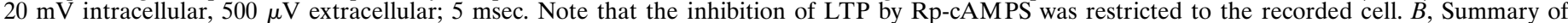

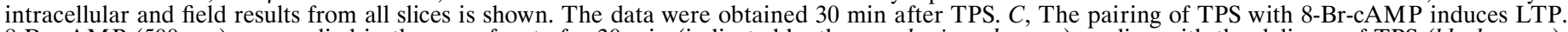

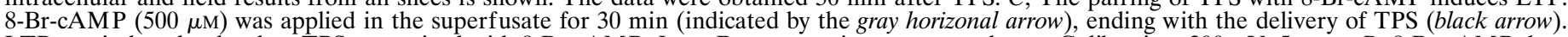

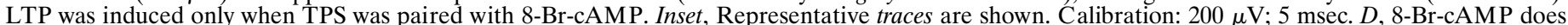

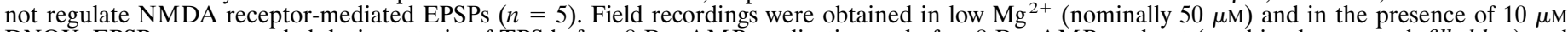

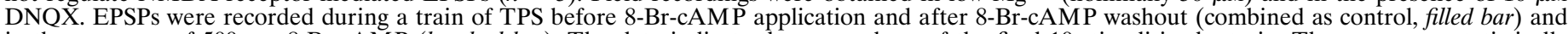

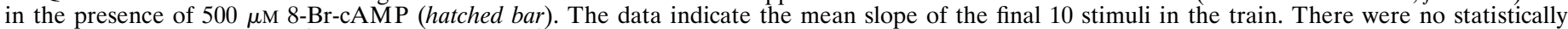
significant group differences (paired $t$ test, $p>0.10$ ). ISO, Isoproterenol.

the postsynaptic cAMP pathway by $\beta$-adrenergic stimulation was necessary for TPS to generate LTP. The effect of Rp-cAMPS, together with the mimicry of the isoproterenol effect by 8 -BrcAMP, indicates that the only contribution of $\beta$-adrenergic stimulation to TPS-LTP is the activation of the postsynaptic cAMP pathway.

$\mathrm{Ca}^{2+}$ influx through NMDA channels is required for the induction of LTP by a variety of stimulus protocols (Larson and Lynch, 1988; Perkel et al., 1993; English and Sweatt, 1996; Thomas et al., 1996). The previously identified role of cAMP in the induction of LTP - as an inhibitor of postsynaptic PP1 activity - places it downstream of $\mathrm{Ca}^{2+}$ influx. However, there are reports of cAMPdependent regulation of NMDA channels (Raman et al., 1996; Westphal et al., 1999) and increased $\mathrm{Ca}^{2+}$ entry through NMDA channels would be likely to promote LTP. To test whether cAMPdependent NMDA receptor regulation contributes to TPS-LTP, the NMDA component of the EPSP was isolated by recording in a low- $\mathrm{Mg}^{2+}$ solution containing the AMPA receptor antagonist DNQX $(10 \mu \mathrm{M})$. TPS was delivered to the slices in the presence or absence of $500 \mu \mathrm{M}$ 8-Br-cAMP. The mean amplitude of the final 10 field EPSPs was determined, because any effect of accelerated recovery of NMDA conductance should be most pronounced late in the TPS train. 8-Br-cAMP had no significant effect on the NMDA-mediated EPSP (Fig. 1D), suggesting that the enhancement of NMDA-mediated currents by cAMP is not likely to play a major role in TPS-induced LTP.

In the mouse hippocampus, TPS-induced LTP requires complex spiking during TPS, an effect that occurs in the absence of $\beta$-receptor activation but that is accentuated and prolonged by isoproterenol (Thomas et al., 1998; Winder et al., 1999). In field recordings obtained during TPS, we found that TPS alone did not evoke complex spikes, or even single spikes (Fig. 2A). However, complex potentials were apparent during TPS when isoproterenol was present. As noted previously (Winder et al., 1999), the negativity of these spikes suggests that they are dendritic potentials, presumably resulting from backpropagation from the soma. Complex waveforms were exhibited within 30 stimuli (the earliest sample obtained during TPS), and the number of discrete potentials 


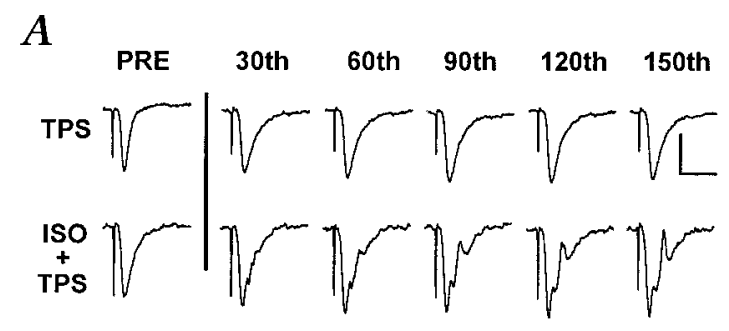

$B$

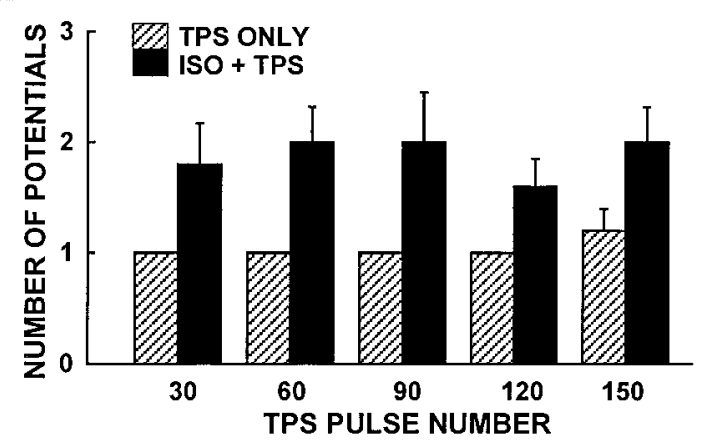

Figure 2. TPS evokes complex potentials when paired with isoproterenol. $A$, Representative field traces recorded immediately before the start of TPS $(P R E)$ and at every 30th stimulus during TPS. In the absence of isoproterenol (top records), no spikes were seen during TPS. The increase in the duration of the EPSP relative to the baseline was typically observed in this experiment. When isoproterenol was present (bottom traces), the unitary EPSP was replaced by a complex waveform, which included spike potentials. Calibration: $500 \mu \mathrm{V} ; 10 \mathrm{msec}$. $B$, Summary of the number of discrete components in sampled field traces during TPS in the absence (hatched bars; $n=5$ ) and presence ( filled bars; $n=5$ ) of $1 \mu \mathrm{M}$ isoproterenol. The groups differed significantly $(p<0.02)$, and there was no patterning effect during TPS $(F<1)$.

per trace remained quite constant for the remainder of the train (Fig. $2 B$ ). These results indicate that, as with mouse hippocampus, the facilitation of repetitive spiking may be an important factor in the ability of $\beta$-adrenergic stimulation to enable LTP in the rat hippocampus.

\section{Activation of the cAMP pathway suppresses PP1 activity and phosphorylates inhibitor-1}

Previous work from this lab established that the cAMP pathway participates in HFS-induced LTP by inhibiting the activity of PP1 (Blitzer et al., 1998). A piece of evidence suggesting that the cAMP pathway may serve a similar role in TPS-LTP is the ability of phosphatase blockers to mimic the LTP-enabling effect of $\beta$-adrenergic stimulation in this induction protocol (Coussens and Teyler, 1996; Thomas et al., 1996).

To test whether cAMP-mediated phosphatase inhibition might underlie the role of the cAMP pathway in TPS-induced LTP, we first assayed PP1 activity in the CA1 region of slices exposed to 8-Br-cAMP (Fig. $3 A$ ). A reliable reduction in PP1 activity was produced by 8 - $\mathrm{Br}$-cAMP relative to unstimulated controls. This result is consistent with the hypothesis that activation of the postsynaptic cAMP pathway contributes to TPS-induced LTP by inhibiting PP1.

The most likely mechanism for the cAMP-induced decrease in PP1 activity involves inhibitor-1. Biochemical experiments have established that PKA phosphorylates I-1, which is a direct and specific inhibitor of PP1 (Shenolikar, 1994), and this pathway was identified as the mechanism of cAMP-dependent HFS-induced LTP (Blitzer et al., 1998). If the role of the cAMP pathway in TPS-LTP is mediated by the I- 1 mechanism, then $\beta$-adrenergic receptor agonists and cAMP pathway activators should increase I-1 phosphorylation in the CA1 region. To test this prediction, isoproterenol or 8-Br-cAMP was applied to hippocampal slices, followed by determination of total and phosphorylated I-1 in CA1. Both
A

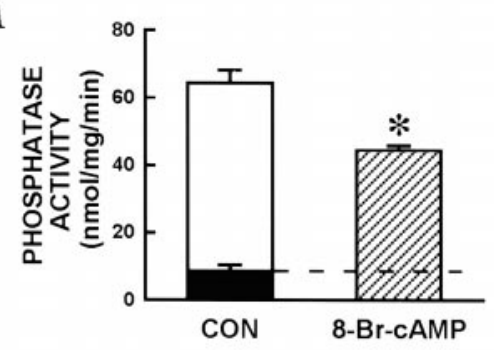

$B$

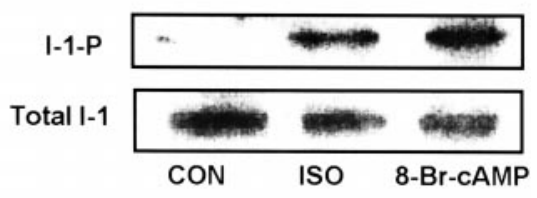

$C_{1}$

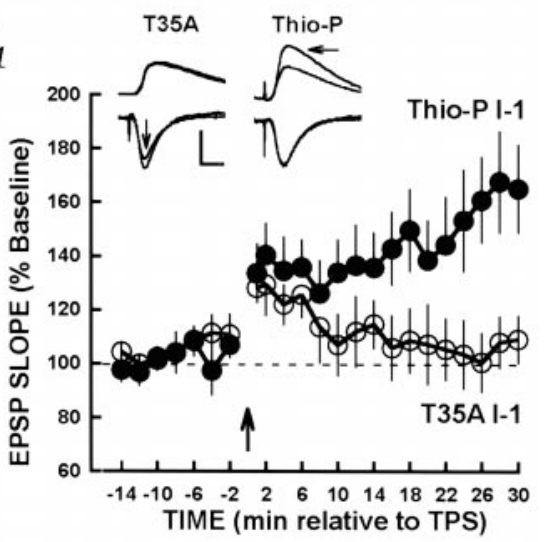

$C_{2}$

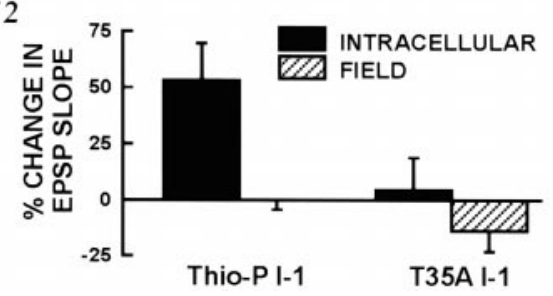

Figure 3. The role of protein phosphatase-1 in TPS-induced LTP. A, Protein phosphatase activity in CA1 is inhibited by activation of the cAMP pathway. In slices exposed to $1 \mathrm{mM} 8$-Br-cAMP for $30 \mathrm{~min}$, total phosphatase activity was significantly lower than that in untreated controls $(p<0.05)$. Similar results were obtained in two independent experiments. The selectivity of the assay for PP1 is shown by the ability of $100 \mathrm{~nm}$ thiophosphorylated inhibitor-1 (I-1-P) to block $>85 \%$ of total phosphatase activity, indicated by the black bar and dashed line. $B$, Endogenous protein phosphatase inhibitor-1 is phosphorylated by stimulation of the cAMP pathway. Tissue homogenates were probed with a monoclonal antibody recognizing either the phosphorylated form of inhibitor-1 selectively (top gel) or both the phosphorylated and nonphosphorylated inhibitor-1 (bottom gel ). Isoproterenol $(10 \mu \mathrm{M})$ and $8-\mathrm{Br}$ cAMP $(500 \mu \mathrm{M})$ increased the levels of $\mathrm{Thr}^{35}$-phosphorylated I-1 4.04 ( 2.28 )-fold and 2.75 ( \pm 0.62$)$-fold, respectively, relative to unstimulated tissue and normalized for total I-1 levels. Both isoproterenol and 8-Br-cAMP increased the level of thiophosphorylated inhibitor-1 (top gel) without significantly changing the total amount of inhibitor-1. Similar results were obtained in two other experiments. $C$, Postsynaptic inhibition of PP1 mimics cAMP pathway activation in TPS-LTP. $C_{1}$, The time course of LTP induced by TPS paired with thiophosphorylated I-1 is shown. The time of TPS is indicated by the arrow. Thiophosphorylated I-1 (Thio-P I-1; $10 \mu \mathrm{M} ; n=9$ ) or inactive, nonphosphorylatable I-1 (T35A I-1; $10 \mu \mathrm{M} ; n=8)$ was applied in the intracellular electrode. A slowly developing LTP was induced by TPS only when thiophosphorylated I-1 was present, with the two groups differing significantly over the last three time points of the experiment (ANOVA, $p<$ 0.05). Inset, Sample intracellular and field traces (top and bottom panels, respectively) from a $T 35 A I-1$ experiment (left) and a Thio- $P$ I- 1 experiment (right) are shown. Presentation details are as described in Figure 1. No field LTP was obtained in any of the slices in this experiment. Calibration: $10 \mathrm{mV}$ intracellular, $250 \mu \mathrm{V}$ extracellular; $5 \mathrm{msec}$. $C_{2}$, Summary data of intracellular and field data at $30 \mathrm{~min}$ after TPS are shown. The intracellular data were derived from the experiment shown in $\mathrm{C}_{1}$. $C O N$, Control. 

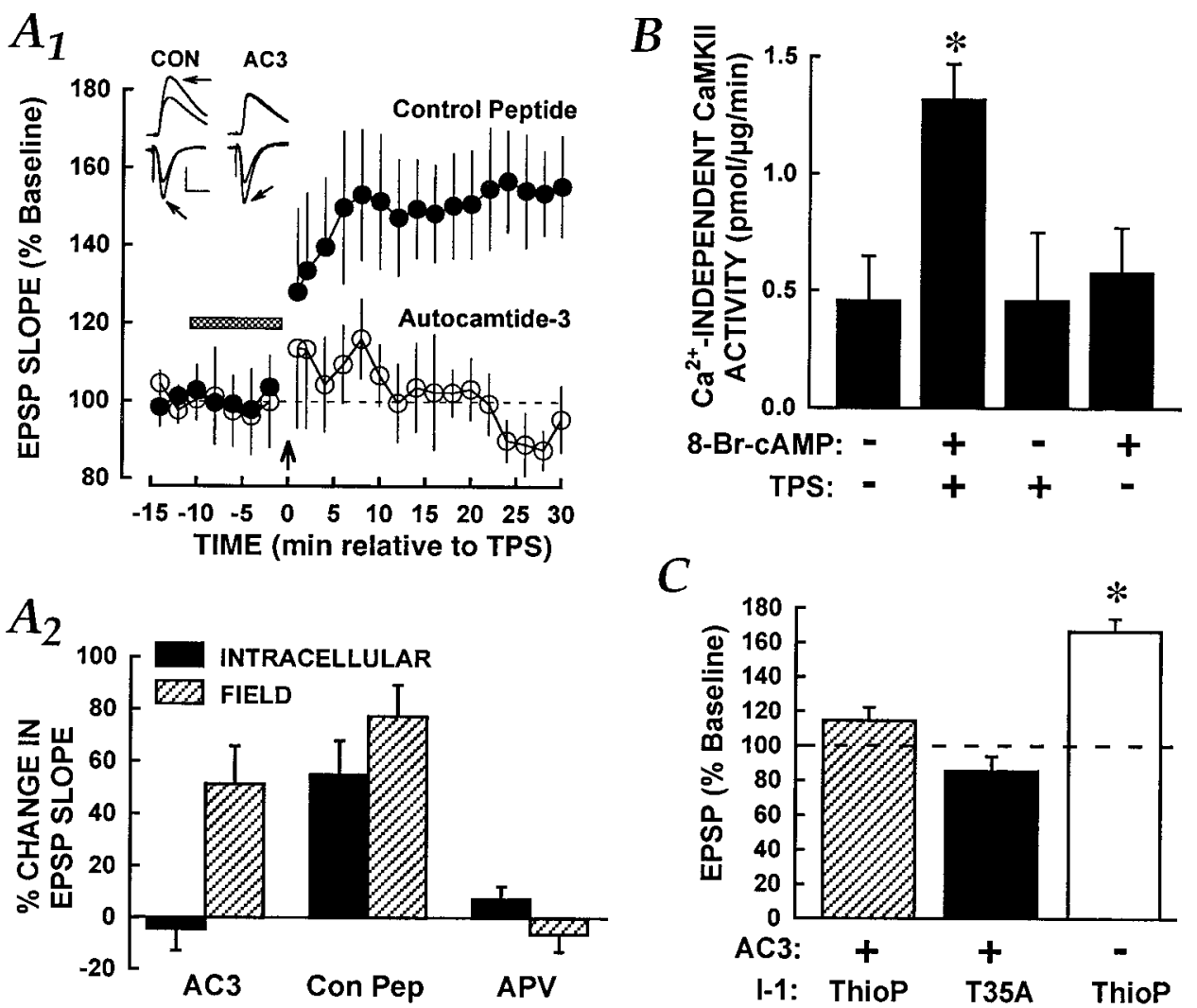

Figure 4. CaMKII integrates TPS and cAMP pathway stimulation. $A$, Postsynaptic CaMKII activity is required for TPS-LTP. $A_{1}$, Time course graph of intracellular EPSPs is shown. The horizontal bar indicates isoproterenol application, and the arrow near the $x$-axis shows the time of TPS. The intracellular electrode contained either inactive control peptide ( $5 \mathrm{mM}$; filled symbols; $n=5)$ or the CaMKII inhibitor autocamtide-3 (AC3; 5 mM; open symbols; $n=5$ ). Normal LTP was obtained in the control cells, but LTP was absent in cells injected with $A C 3$. The groups differed significantly over the last three time points (ANOVA, $p<0.01$ ). Inset, Representative intracellular and field EPSPs from a control peptide experiment $(l e f t)$ and from an $A C 3$ experiment (right) are shown. Presentation details are as described in Figure 1. Calibration: $10 \mathrm{mV}$ intracellular, $250 \mu \mathrm{V}$ extracellular; $10 \mathrm{msec} . A_{2}$, Summary graph of data recorded at $30 \mathrm{~min}$ after TPS is shown. The filled bars represent changes in intracellular synaptic strength, and the hatched bars represent field data. Con Pep, Peptide control for $A C 3$. The only group to exhibit intracellular LTP was the combined TPS plus Con Pep group, which was significantly different from both other groups (Newman-Keuls test, $p$ values $<0.05$ ). $B$, TPS paired with 8 -Br-cAMP $\left(500 \mu M\right.$ ) increases Ca ${ }^{2+}-$ independent CaMKII activity. Similar results were obtained in three independent experiments, one of which is shown here. The slices that were exposed to the combination treatment showed significantly greater CaMKII activity in the absence of $\mathrm{Ca}^{2+}$ than did all other groups (Newman-Keuls test, ${ }^{*} p<$ 0.05 ), which did not differ among themselves. No group differences in total CaMKII activity were observed (ANOVA, $p>0.20 ;$ mean activity $=3.29 \pm$ $0.39 \mathrm{pmol} \cdot \mu \mathrm{g}^{-1} \cdot \mathrm{min}^{-1}$ pooled across groups). $C$, The blockade of LTP by a CaMKII inhibitor is resistant to PP1 suppression. Summary of results from experiments in which LTP was induced using TPS, with data taken from the final three time points (26-30 min after TPS), is shown. Substances were applied in the intracellular recording electrode. Autocamtide-3 $(2.5 \mathrm{~mm})$ was combined either with nonphosphorylatable T35A I- 1 (T35A; filled bar; $n=$ 6 ) or with thiophosphorylated I-1 (ThioP; hatched bar; $n=6)$. In other cells, thiophosphorylated I-1 was presented with the control peptide for autocamtide-3 (2.5 mM; open bar; $n=6)$. The asterisk indicates a significant increase in EPSP slope above baseline (ANOVA, $p<0.05)$.

isoproterenol and 8-Br-cAMP increased $\mathrm{Thr}^{35}$-phosphorylated I-1 levels, by fourfold and threefold, respectively (Fig. 3B).

Inhibitor-1 substitutes for the cAMP pathway in TPS-LTP

If phosphorylation of I-1 mediates the isoproterenol effect in TPSLTP, then postsynaptic application of phosphorylated I-1 should mimic the effect of isoproterenol by enabling TPS to induce LTP. When cells were recorded with electrodes containing recombinant, constitutively active thiophosphorylated I-1, TPS alone was sufficient to induce LTP (Fig. $3 C ; 165 \pm 16 \%$ at $30 \mathrm{~min}$ ). In control cells injected with the nonphosphorylatable T35A mutant of I-1, TPS induced only a transient potentiation, which returned to baseline within $10 \mathrm{~min}(109 \pm 9 \%$ at $30 \mathrm{~min})$. Thiophosphorylated I-1 had no effect on the EPSP when TPS was omitted $(103 \pm 11 \%$ at the corresponding time point; $n=3$ ). The ability of activated I- 1 to substitute for $\beta$-adrenergic stimulation provides strong support for I-1 as the mediator of the cAMP pathway contribution to TPS-LTP.

\section{CaMKII integrates TPS and the cAMP pathway in TPS-induced LTP}

Many studies have identified CaMKII as an essential component of the LTP-signaling pathway, at least during the induction phase (Lledo et al., 1995; Otmakhov et al., 1997). The induction of LTP by trains of HFS is accompanied by increased $\mathrm{Thr}^{286}$ phosphorylation of CaMKII and the generation of $\mathrm{Ca}^{2+}$-independent CaMKII activity (Malinow et al., 1989; Fukunaga et al., 1995; Blitzer et al., 1998). CaMKII is a substrate for PP1 (Strack et al., 1997), and the cAMP-regulated phosphatase gate described for HFS-induced LTP acts directly on CaMKII (Blitzer et al., 1998).

The role of CaMKII in TPS-LTP and its possible interaction with cAMP-sensitive phosphatase activity were explored in a series of experiments. First, we determined whether postsynaptic CaMKII activity is required for TPS-LTP by recording from cells with electrodes filled with either the selective CaMKII inhibitor autocamtide- 3 or an inactive control peptide (Braun and Schulman, 1995). Autocamtide-3 blocks CaMKII and protein kinase $\mathrm{C}$ with $\mathrm{IC}_{50}$ values of $\sim 3$ and $\sim 500 \mu \mathrm{M}$, respectively. On the basis of previous work with inhibitors introduced through intracellular electrodes (Hvalby et al., 1994; Blitzer et al., 1995), we estimate that our electrode concentration of $5 \mathrm{~mm}$ yielded an intracellular concentration of $\sim 50 \mu \mathrm{M}$, which is expected to effectively block CaMKII with little effect on protein kinase C. TPS paired with isoproterenol induced LTP in the cells injected with the control peptide, but no LTP was seen in cells recorded with autocamtide-3 (Fig. 4A). In addition, LTP was blocked by the bath-applied NMDA antagonist APV (Fig. 4A2). These results identify 
CaMKII as a necessary signaling component in TPS-LTP and suggest that, in common with several other forms of LTP, CaMKII is activated by $\mathrm{Ca}^{2+}$ influx through NMDA channels.

We next asked whether the stimulation of the cAMP pathway could enable TPS-LTP by activating CaMKII. Total and $\mathrm{Ca}^{2+}$ independent $\mathrm{CaMKII}$ activities were measured in homogenates from area CA1 of slices exposed to either TPS, 8-Br-cAMP, or both. Only the combination treatment increased $\mathrm{Ca}^{2+}$. independent CaMKII activity in the CA1 region above the level observed in unstimulated control slices (Fig. 4B). $\mathrm{Ca}^{2+}$. independent CaMKII activity was not increased above basal by treatment of the slices with 8-Br-cAMP or TPS alone. None of the groups differed with respect to total CaMKII activity. Thus, the detection of coincident TPS and $\beta$-adrenergic activity, which induces LTP, is complete at the level of CaMKII.

The increase in constitutive CaMKII activity, restricted to slices receiving both TPS and $8-\mathrm{Br}-\mathrm{cAMP}$, is consistent with the expected behavior of the phosphatase gate acting on CaMKII. TPS alone should activate the CaMKII pathway [probably to a submaximal level at $10 \mathrm{~Hz}$; see Coomber (1998)] and produce a calcineurin-dependent increase in the activity of PP1, which would be free to dephosphorylate CaMKII. The application of $8-\mathrm{Br}$ cAMP alone should inhibit PP1, but in the absence of $\mathrm{Ca}^{2+}$ influx provided by TPS, no CaMKII activation will occur. Thus, the gating model predicts an increase in $\mathrm{Ca}^{2+}$-independent $\mathrm{CaMKII}$ activity only after combined TPS and cAMP pathway stimulation, the former contributing $\mathrm{Ca}^{2+}$ for CaMKII autophosphorylation and the latter protecting the nascent autonomous CaMKII activity by phosphorylating I-1 and suppressing PP1. This model places PP1 upstream of CaMKII and predicts that phosphatase inhibition should not overcome the blockade of TPS-LTP by a CaMKII inhibitor. We tested this prediction by blocking postsynaptic CaMKII with autocamtide-3 and stimulating the cell with combined TPS and isoproterenol. This treatment blocked LTP even when PP1 was directly inhibited by the postsynaptic injection of thiophosphorylated I-1 (Fig. 4C). The requirement for CaMKII activity despite such aggressive inhibition of PP1 clearly indicates that CaMKII is downstream of PP1.

\section{TPS-induced LTP does not require increased postsynaptic excitability}

The induction of LTP by synaptic stimulation requires the pairing of presynaptic activity with postsynaptic depolarization (Bliss and Collingridge, 1993). The source of the depolarization is usually considered to be the summation of EPSPs, particularly those mediated by NMDA channels. A potential additional source of dendritic depolarization is the backpropagation of action potentials from the soma, which could contribute to LTP [Spruston et al. (1995); Magee and Johnston (1997); but see Gustafsson et al. (1987) for evidence that postsynaptic spiking does not contribute to LTP]. As seen in Fig. 2 and as observed in experiments on mouse hippocampus (Thomas et al., 1998; Winder et al., 1999), spiking activity can occur during a train of TPS.

Is an increase in postsynaptic excitability sufficient to induce LTP in rat hippocampus? We examined this issue by comparing spiking during TPS in cells injected with either thiophosphorylated inhibitor-1 or inactive T35A inhibitor-1. In preliminary experiments, a stimulus intensity adequate to induce LTP, which produced the complex field potentials shown in Figure 2, failed to reliably induce multiple action potentials in the recorded postsynaptic neuron during TPS. To obtain repetitive postsynaptic firing during TPS, the stimulus intensity had to be increased to a level sufficient for test pulses to elicit single action potentials (Fig. 5A). However, a comparison of the cells injected with Thio-P I-1 and T35A I-1 showed no differences in the number of action potentials evoked by stimuli during TPS, despite a clear group difference in the induction of LTP (Fig. 5A,B). Thus, repetitive postsynaptic firing was not sufficient to enable TPS-induced LTP, but the additional blockade of postsynaptic PP1 produced a lasting potentiation. In addition, because thiophosphorylated I-1 did not increase
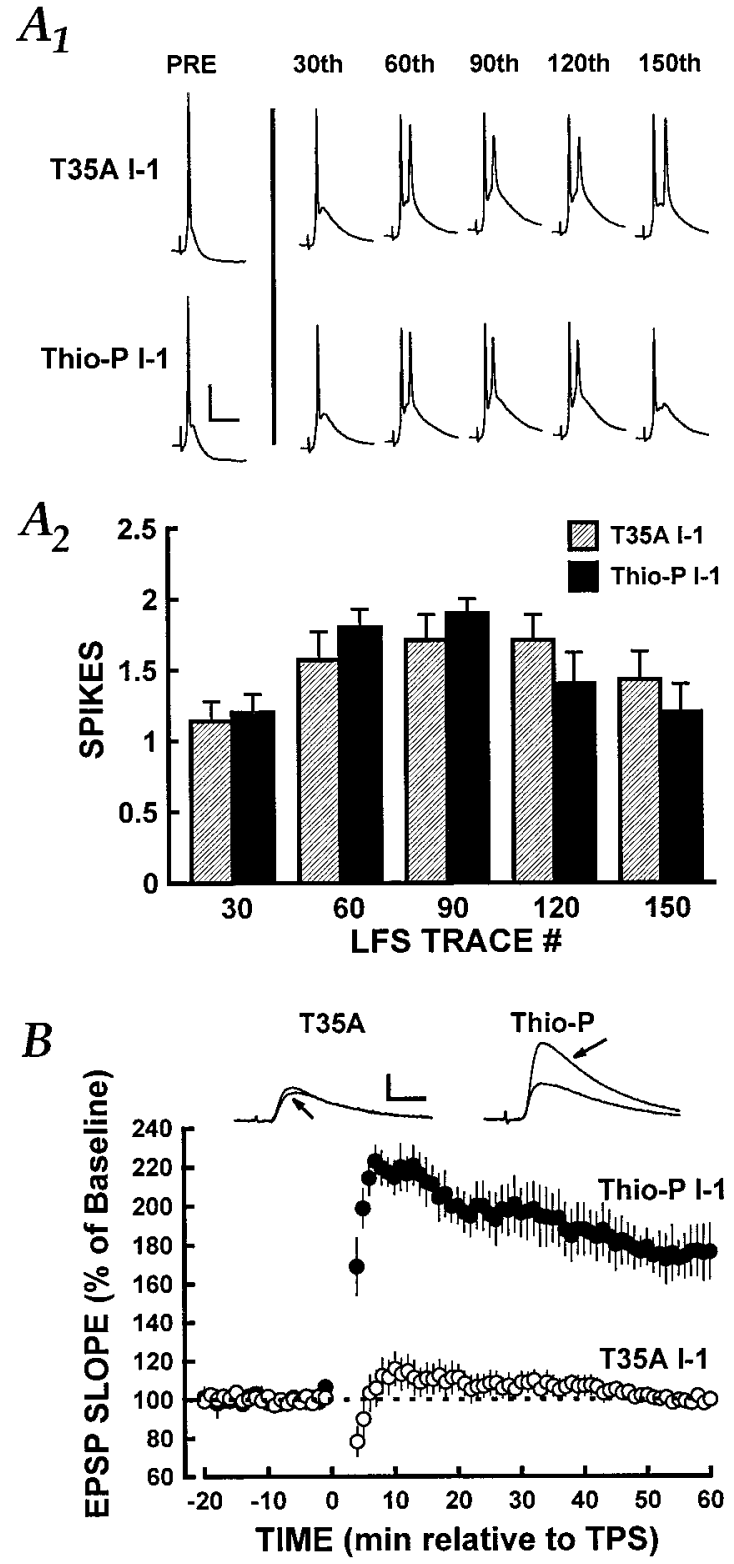

Figure 5. The direct inhibition of postsynaptic PP1 enables TPS-induced LTP without an increase in postsynaptic spiking. $A$, Postsynaptic activity during TPS was not increased by thiophosphorylated I-1. $A_{1}$, Samples of postsynaptic potentials recorded just before TPS (PRE) and at every 30th stimulus during TPS are shown. Double spikes were evoked during TPS in both groups. Of all TPS samples recorded $(n=85)$, only one stimulus produced three spikes, with all of the others yielding one or two. Calibration: $10 \mathrm{mV} ; 10 \mathrm{msec}$. $A_{2}$, Summary of the number of spikes produced by stimuli during TPS is shown. There were no statistically significant differences between cells recorded with thiophosphorylated I-1 and those recorded with T35A I-1. A patterning effect was obtained, with the number of spikes at trace 90 significantly higher than those at traces 30 and 150 (Newman-Keuls, $p<0.01$ and 0.05 , respectively). $B$, TPS induces LTP when paired with intracellular application of thiophosphorylated I-1. Only cells impaled with Thio-PI-1 $(n=10)$ showed LTP after TPS. The rapid induction of LTP compared with that shown in Figure 3 is probably caused by the stronger stimulation used during TPS (see Materials and Methods). No lasting effect on synaptic efficiency was seen when TPS was delivered to cells impaled with electrodes containing inactive T35A I-1 $(n=7)$. The inset shows sample EPSPs obtained during the baseline period and at $60 \mathrm{~min}$ after TPS.

membrane excitability, the induction of burst firing by isoproterenol must be independent of the inhibition of postsynaptic PP1 by the cAMP pathway.

\section{DISCUSSION}

The present paper addresses some of the biochemical mechanisms that underlie LTP induced by $\theta$ frequency stimulation in combina- 
tion with activation of the postsynaptic cAMP pathway. From the standpoint of the widely proposed relationship between synaptic plasticity and behavioral formation, the study of LTP induced by this pattern of stimulation should prove valuable. Hippocampusbased memory is likely to be better modeled by TPS-induced LTP than by the HFS protocols more typically used, because prolonged $100 \mathrm{~Hz}$ trains do not occur within natural in vivo firing patterns (Fenton and Muller, 1998; Dobrunz and Stevens, 1999). Rather, hippocampal activity recorded in behaving animals consists of short bursts of spikes interspersed with relatively long, quiescent intervals. The median frequency of the spikes within a burst, adjusted to $31^{\circ} \mathrm{C}$, is $\sim 11 \mathrm{~Hz}$. The bursts are short ( $<15$ pulses), and the interburst intervals are in the order of $0.7 \mathrm{sec}$. Dobrunz and Stevens (1999) have shown that such stimulation patterns, applied for 256 pulses, can induce LTP. However, our data and those of others (Thomas et al., 1996; Katsuki et al., 1997; Winder et al., 1999) suggest that the probability of LTP induction will be enhanced by coactivation of $\beta$-adrenergic receptors. Hippocampus activity in the $\theta$ range, coupled with locus ceruleus stimulation (Foote et al., 1983; Aston-Jones et al., 1994), should provide an effective stimulus for modifying synaptic efficiency.

TPS-induced LTP has been studied in detail in mouse hippocampal slices. However, the induction conditions and underlying mechanisms of TPS-induced LTP appear to be quite different from those observed in rat hippocampus. In mouse slices, a train of 150 stimuli delivered at $5 \mathrm{~Hz}$ induces LTP, whereas longer trains cause depotentiation (Thomas et al., 1996). These phenomena are linked to complex spike bursting that occurs during the first 150 pulses, followed by predominantly single spikes in response to subsequent stimuli. When isoproterenol is applied to the mouse hippocampus, the longer trains persist in eliciting complex spikes, and depotentiation is inhibited (Thomas et al., 1998). In contrast, in rat hippocampus, 150 pulses delivered at $10 \mathrm{~Hz}$ do not cause LTP on their own, nor do they evoke complex spike bursting. Furthermore, the inhibition of postsynaptic PP1 enables TPS to induce LTP without any increase in postsynaptic excitability. This finding does not exclude a role for postsynaptic burst firing in the induction of TPS-LTP; however, it indicates that some other process, which is inhibited by PP1 activity, contributes to TPS-LTP in the rat hippocampus. Our data suggest that this process is the regulation of postsynaptic CaMKII activity.

Studies that used widely spaced trains of HFS in the rat CA3 $\rightarrow$ CA1 synapse have shown the importance of the indirect regulation of CaMKII by the cAMP pathway in LTP at the rat CA3 $\rightarrow$ CA1 synapse (Blitzer et al., 1995, 1998). The LTP-induction model based on those results included a signaling pathway and a PP1-regulated gate, each incorporating $\mathrm{Ca}^{2+} /$ calmodulin-dependent enzymes. The signaling pathway is activated by CaMKII, and the position of the gate is determined by the balance of adenylyl cyclase activity and calcineurin activity. When synaptic stimulation is relatively strong, such as that used in the spaced HFS experiments, high levels of $\mathrm{Ca}^{2+} /$ calmodulin are attained that are sufficient to activate the signaling pathway (because of CaMKII activity) and keep the gate open (because of adenylyl cyclase activity and consequent PP1 inhibition).

How can this model handle LTP induction by weak synaptic stimulation paired with $\beta$-adrenergic stimulation? The abilities of different patterns of stimulation to activate calcineurin and adenylyl cyclase may offer a solution. Weak stimulation, exemplified by lowfrequency stimulation of $1 \mathrm{~Hz}$, can induce LTD in area CA1 via a calcineurin-dependent process (Mulkey et al., 1994). This effect is blocked by 8 -Br-cAMP, confirming an antagonistic relationship between calcineurin and the cAMP pathway and indicating that such weak stimulation preferentially activates calcineurin. Much stronger synaptic stimulation is needed to activate the cAMP pathway fully; e.g., widely spaced triple trains of HFS are sufficient, but a single HFS train is not (Frey et al., 1993; Blitzer et al., 1995). With weak stimulation, the closed position of the gate would be favored, because calcineurin is not opposed by the cAMP pathway and PP1 is active. The concurrent activation of the cAMP pathway by some other method, such as $\beta$-adrenergic stimulation, would open the PP1 gate and permit CaMKII-dependent signaling.

The application of the gating model to TPS-induced LTP is supported by the results presented in this paper. These include the blockade of TPS-LTP by inhibitors of the NMDA receptor, of CaMKII, and of PKA, the phosphorylation of endogenous I- 1 by TPS paired with either isoproterenol or 8-Br-cAMP, the ability of postsynaptic thiophosphorylated I-1 to substitute for $\beta$-adrenergic stimulation, and the stimulation of $\mathrm{Ca}^{2+}$-independent $\mathrm{CaMKII}$ activity by TPS and 8-Br-cAMP applied together but not separately. It thus appears that two forms of cAMP-dependent LTP, one induced by strong synaptic stimulation and the other by more physiologically relevant stimulation coupled with an active postsynaptic cAMP pathway, use similar mechanisms.

The gate-related effect of the cAMP pathway on CaMKII is independent of the ability of isoproterenol to enhance burst firing during TPS (Fig. 1) (Winder et al., 1999), because direct postsynaptic inhibition of PP1 did not increase excitability during TPS (Fig. 5). However, these two cAMP-dependent processes could act in concert to strengthen postsynaptic CaMKII signaling. The backpropagation of even individual action potentials produces dendritic $\mathrm{Ca}^{2+}$ transients in CA1 neurons (Callaway and Ross, 1995; Spruston et al., 1995), and burst firing during a train of TPS might be expected to substantially increase the $\mathrm{Ca}^{2+}$ signal. An additional contribution of repetitive firing to $\mathrm{Ca}^{2+}$ influx is suggested by the recent demonstration of a postsynaptic $\mathrm{Na}^{+}$-dependent increase in NMDA receptor-mediated currents (Yu and Salter, 1998). Under some conditions, such as in the mouse hippocampus, the rise in dendritic $\mathrm{Ca}^{2+}$ concentrations produced by bursting may be sufficient to enable TPS-induced LTP, perhaps by activating $\mathrm{Ca}^{2+}$. dependent adenylyl cyclases. The $\mathrm{Ca}^{2+}$ entry that accompanies widely spaced trains of HFS increases postsynaptic cAMP in this manner (Blitzer et al., 1995), and the backpropagation of bursts into the dendrites during TPS may similarly activate the cAMP pathway. However, our data in the rat hippocampus show that the $\mathrm{Ca}^{2+}$ entry associated with TPS-induced bursting is not adequate to enable LTP, and additional inhibition of postsynaptic PP1 activity is necessary. It seems reasonable that, under physiological conditions, cAMP-dependent processes are activated to a lesser degree than that achieved in slice experiments, so that both burst firing and phosphatase inhibition may contribute to the role of the cAMP pathway in LTP and memory.

\section{REFERENCES}

Aston-Jones G, Rajkowski J, Kubiak P, Alexinsky T (1994) Locus coeruleus neurons in monkey are selectively activated by attended cues in a vigilance task. J Neurosci 14:4467-4480.

Bliss TV, Collingridge GL (1993) A synaptic model of memory: long-term potentiation in the hippocampus. Nature 361:31-39.

Blitzer RD, Wong T, Nouranifar R, Iyengar R, Landau EM (1995) Postsynaptic cAMP pathway gates early LTP in hippocampal CA1 region. Neuron 15:1403-1414.

Blitzer RD, Connor JH, Brown GP, Wong T, Shenolikar S, Iyengar R, Landau EM (1998) Gating of CaMKII by cAMP-regulated protein phosphatase activity during LTP. Science 280:1940-1942.

Braun AP, Schulman H (1995) A non-selective cation current activated via the multifunctional $\mathrm{Ca}^{2+}$-calmodulin-dependent protein kinase in human epithelial cells. J Physiol (Lond) 488:37-55.

Callaway JC, Ross WN (1995) Frequency-dependent propagation of sodium action potentials in dendrites of hippocampal CA1 pyramidal neurons. J Neurophysiol 74:1395-1403.

Coomber C (1998) Current theories of neuronal information processing performed by $\mathrm{Ca} 2+/$ calmodulin-dependent protein kinase II with support and insights from computer modelling and simulation. Comput Chem 22:251-263.

Coussens CM, Teyler TJ (1996) Protein kinase and phosphatase activity regulate the form of synaptic plasticity expressed. Synapse 24:97-103.

Dobrunz LE, Stevens CF (1999) Response of hippocampal synapses to natural stimulation patterns. Neuron 22:157-166.

Dudek SM, Bear MF (1992) Homosynaptic long-term depression in area CA1 of hippocampus and effects of $N$-methyl-D-aspartate receptor blockade. Proc Natl Acad Sci USA 89:4363-4367.

English JD, Sweatt JD (1996) Activation of p42 mitogen-activated protein kinase in hippocampal long term potentiation. $J$ Biol Chem 271:24329-24332.

Fenton AA, Muller RU (1998) Place cell discharge is extremely variable 
during individual passes of the rat through the firing field. Proc Natl Acad Sci USA 95:3182-3187.

Foote SL, Bloom FE, Aston-Jones G (1983) Nucleus locus ceruleus: new evidence of anatomical and physiological specificity. Physiol Rev 63:844-914.

Frey U, Huang YY, Kandel ER (1993) Effects of cAMP simulate a late stage of LTP in hippocampal CA1 neurons. Science 260:1661-1664.

Fukunaga K, Muller D, Miyamoto E (1995) Increased phosphorylation of $\mathrm{Ca}^{2+} /$ calmodulin-dependent protein kinase II and its endogenous substrates in the induction of long-term potentiation. J Biol Chem 270:6119-6124.

Gustafsson B, Wigstrom H, Abraham WC, Huang YY (1987) Long-term potentiation in the hippocampus using depolarizing current pulses as the conditioning stimulus to single volley synaptic potentials. J Neurosci 7:774-780.

Hvalby O, Hemmings Jr HC, Paulsen O, Czernik AJ, Nairn AC, Godfraind JM, Jensen V, Raastad M, Storm JF, Andersen P, Greengard P (1994) Specificity of protein kinase inhibitor peptides and induction of long-term potentiation. Proc Natl Acad Sci USA 91:4761-4765.

Katsuki H, Izumi Y, Zorumski CF (1997) Noradrenergic regulation of synaptic plasticity in the hippocampal CA1 region. J Neurophysiol 77:3013-3020.

Larson J, Lynch G (1988) Role of $N$-methyl-D-aspartate receptors in the induction of synaptic potentiation by burst stimulation patterned after the hippocampal theta-rhythm. Brain Res 441:111-118.

Lisman J (1994) The CaM kinase II hypothesis for the storage of synaptic memory. Trends Neurosci 17:406-412.

Lledo PM, Hjelmstad GO, Mukherji S, Soderling TR, Malenka RC, Nicoll RA (1995) Calcium/calmodulin-dependent kinase II and long-term potentiation enhance synaptic transmission by the same mechanism. Proc Natl Acad Sci USA 92:11175-11179.

Ma L, Zablow L, Kandel ER, Siegelbaum SA (1999) Cyclic AMP induces functional presynaptic boutons in hippocampal CA3-CA1 neuronal cultures. Nat Neurosci 2:24-30.

Madison DV, Nicoll RA (1986) Cyclic adenosine 3',5'-monophosphate mediates $\beta$-receptor actions of noradrenaline in rat hippocampal pyramidal cells. J Physiol (Lond) 372:245-259.

Magee JC, Johnston D (1997) A synaptically controlled, associative signal for Hebbian plasticity in hippocampal neurons. Science 275:209-213.

Malenka RC, Nicoll RA (1999) Long-term potentiation-a decade of progress? Science 285:1870-1874.

Malinow R, Schulman H, Tsien RW (1989) Inhibition of postsynaptic PKC or CaMKII blocks induction but not expression of LTP. Science 245:862-866.

Mayford M, Wang J, Kandel ER, O’Dell TJ (1995) CaMKII regulates the frequency-response function of hippocampal synapses for the production of both LTD and LTP. Cell 81:891-904

Mulkey RM, Endo S, Shenolikar S, Malenka RC (1994) Involvement of a calcineurin/inhibitor-1 phosphatase cascade in hippocampal long-term depression. Nature 369:486-488.

Otmakhov N, Griffith LC, Lisman JE (1997) Postsynaptic inhibitors of calcium/calmodulin-dependent protein kinase type II block induction but not maintenance of pairing-induced long-term potentiation. J Neurosci 17:5357-5365.

Otmakhova NA, Otmakhov N, Mortenson LH, Lisman JE (2000) Inhibition of the cAMP pathway decreases early long-term potentiation at CA1 hippocampal synapses. J Neurosci 20:4446-4451.

Pedarzani P, Storm JF (1993) PKA mediates the effects of monoamine transmitters on the $\mathrm{K}^{+}$current underlying the slow spike frequency adaptation in hippocampal neurons. Neuron 11:1023-1035.

Perkel DJ, Petrozzino JJ, Nicoll RA, Connor JA (1993) The role of $\mathrm{Ca}^{2+}$ entry via synaptically activated NMDA receptors in the induction of long-term potentiation. Neuron 11:817-823.

Raman IM, Tong G, Jahr CE (1996) $\beta$-Adrenergic regulation of synaptic NMDA receptors by cAMP-dependent protein kinase. Neuron 16:415-421.

Segal M, Greenberger V, Hofstein R (1981) Cyclic AMP-generating systems in rat hippocampal slices. Brain Res 213:351-364.

Shenolikar S (1994) Protein serine/threonine phosphatases-new avenues for cell regulation. Annu Rev Cell Biol 10:55-86.

Snyder GL, Girault JA, Chen JY, Czernik AJ, Kebabian JW, Nathanson JA, Greengard P (1992) Phosphorylation of DARPP-32 and protein phosphatase inhibitor-1 in rat choroid plexus: regulation by factors other than dopamine. J Neurosci 12:3071-3083.

Spruston N, Schiller Y, Stuart G, Sakmann B (1995) Activity-dependent action potential invasion and calcium influx into hippocampal CA1 dendrites. Science 268:297-300.

Strack S, Barban MA, Wadzinski BE, Colbran RJ (1997) Differential inactivation of postsynaptic density-associated and soluble $\mathrm{Ca}^{2+} /$ calmodulin-dependent protein kinase II by protein phosphatases 1 and 2A. J Neurochem 68:2119-2128.

Thomas MJ, Moody TD, Makhinson M, O’Dell TJ (1996) Activitydependent $\beta$-adrenergic modulation of low frequency stimulation induced LTP in the hippocampal CA1 region. Neuron 17:475-482.

Thomas MJ, Watabe AM, Moody TD, Makhinson M, O'Dell TJ (1998) Postsynaptic complex spike bursting enables the induction of LTP by theta frequency synaptic stimulation. J Neurosci 18:7118-7126.

Westphal RS, Tavalin SJ, Lin JW, Alto NM, Fraser ID, Langeberg LK, Sheng M, Scott JD (1999) Regulation of NMDA receptors by an associated phosphatase-kinase signaling complex. Science 285:93-96.

Winder DG, Martin KC, Muzzio IA, Rohrer D, Chruscinski A, Kobilka B, Kandel ER (1999) ERK plays a regulatory role in induction of LTP by theta frequency stimulation and its modulation by $\beta$-adrenergic receptors. Neuron 24:715-726.

Yu XM, Salter MW (1998) Gain control of NMDA-receptor currents by intracellular sodium. Nature 396:469-474. 